Concept Paper

\title{
Omega-3 fatty acid supplementation - A possible dietary adjunct to enhance immune therapy in cancer?
}

\author{
Nikolaus Wachtel ${ }^{1,2}$, Nadine Rohwer ${ }^{1}$, Anne Pietzner ${ }^{3}$, Andreas Loew ${ }^{3}$, Karsten H. Weylandt ${ }^{1,3, *}$ \\ 1 Medical Department, Division of Gastroenterology, Campus Virchow-Klinikum, Charité University Medicine, Berlin, \\ Germany \\ 2 Division of Hand, Plastic and Aesthetic Surgery, University Hospital, LMU Munich, Germany \\ 3 Medical Department, Division of Gastroenterology, Oncology, Hematology, Rheumatology and Diabetes, Ruppiner \\ Kliniken, Brandenburg Medical School, Neuruppin, Germany \\ * Correspondence: karsten.weylandt@mhb-fontane.de; Tel.: +49-3391-39-3210
}

\section{Abstract}

Omega-3 polyunsaturated fatty acids (n-3 PUFA) have been found to be modulators of immune function. Additionally, they may affect the growth of colorectal cancer (CRC). With the advent of novel treatment approaches in oncology targeting immune checkpoint inhibition and aiming to boost the immune response against tumors the exact role of n-3 and n-6 PUFA in inflammation as well as in CRC needs to be re-evaluated in order to understand potential interactions with these new treatment paradigms. Interestingly, for the cyclooxygenase (COX) inhibitor aspirin a possible synergistic effect together with a PD1-Ligand antibody has been shown. However, could n-3 PUFA be disadvantageous in the context of immune tumor therapy due to an immune suppressive effect that has been described for these fatty acids in the past, or could they also enhance the effect of immune checkpoint inhibition?

In this paper, we discuss the current data regarding the immune modulatory as well as the anti-CRC effect of n-3 PUFA. Arguing towards an immune-activating effect of n-3 PUFA, we demonstrate the results of a pilot study. Here, we show that incubation of human peripheral blood mononuclear cells (PBMCs) with the n-3 PUFA docosahexaenoic acid (DHA) significantly decreases CRC-cell supernatant-triggered secretion of IL-10 and increases secretion of TNF- $\alpha$, while the omega-6 polyunsaturated fatty acid (n-6 PUFA) arachidonic acid (AA) reduced TNF- $\alpha$ secretion. These changes in cytokine secretion upon incubation with DHA demonstrate a possible enhancing effect of n-3 PUFA on an anti-tumor immune response.

Keywords: Omega-3 and omega-6 polyunsaturated fatty acids; colorectal cancer; Cancer Immune Therapy.

Abbreviations: AA, arachidonic acid; ASA, acetylsalicylic acid; CM, conditioned media derived from human colorectal adenocarcinoma HT-29 cells; COX, cyclooxygenase; CRC, colorectal cancer; DHA, docosahexaenoic acid; EPA, eicosapentaenoic acid; LX, lipoxin; n-3, omega-3; n-6, omega-6; NSAID, nonsteroidal anti-inflammatory drug; PBMC, peripheral blood mononuclear cell; PG, prostaglandin; PUFA, polyunsaturated fatty acid; SEM, standard error of the mean; TX, thromboxane. 


\section{1. Introduction}

43 Aspirin and Cancer Immune Therapy in Colorectal Cancer

44

45

46

47

48

49

50

51

52
Colorectal cancer (CRC) is one of the most common cancers worldwide [1, 2]. Several epidemiological studies show that the long-term intake of acetylsalicylic acid (ASA, aspirin) and other NSAIDs reduces the incidence of CRC [3-7]. Moreover, aspirin-intake is associated with improved overall survival in CRC-patients [8,9]. These beneficial effects are associated with the reduced conversion of the omega-6 polyunsaturated fatty acid (n-6 PUFA) arachidonic acid (AA) into biologically active eicosanoids $[5,10]$. Experimental evidence suggests that CRC and other tumors evade immune surveillance and T-cell mediated immune response by an overproduction of prostaglandin E2 (PGE2) [11, 12]. PGE2 is established as pro-tumorigenic eicosanoid in CRC development [13-15] and oral treatment with aspirin decreases colonic tissue PGE2 levels in healthy individuals [7] and in those suffering from CRC [16]. Thus, NSAIDs, such as aspirin, can help reverse immune evasion by reducing high levels of anti-inflammatory, tumor-promoting lipid mediators, such as PGE2 [15, 17-21].

Recently, several studies have been published, which offer the prospect to a novel treatment of CRC by targeting cancer-immune checkpoints, such as the programmed cell death 1 (PD-1) pathway, in the tumor microenvironment [12]. The PD-1 pathway is a negative feedback system that suppresses the Th1 cytotoxic immune response. Its blockade with the PD-1 monoclonal antibody pembrolizumab can overcome immune resistance of tumor cells [22, 23]. In this context, Le et al. and others demonstrated that especially CRC with high-level microsatellite instability (MSI) are susceptible to the blockade of the PD-1 pathway [12, 22, 24, 25]. Indeed, the U.S. Food and Drug Administration (FDA) approved the anti-PDCD1 (PD-1) antibody pembrolizumab for treating solid tumors with high-level microsatellite instability (MSI) or mismatch repair deficiency, including MSI-high CRC. These have a large number of neoantigens due to a high number of (frameshift) mutations. Particularly for this CRC subgroup the concept of an anti-tumor immune response is well represented [26, 27]. The inflammatory reaction in the tumor-microenvironment is thought to represent the host's local immune response against infiltrating tumor cells [28]. Numerous studies concluded that the inflammatory infiltrate in and around the tumor correlates with improved survival [28-30]. Especially tumor infiltrating $\mathrm{T}$ lymphocytes and their subsets show strong associations with disease outcome. Thus, a pronounced infiltrate of cells positive for T-cell markers such as CD3, CD45RO (memory T-lymphocytes), and CD8 (cytotoxic T-lymphocytes) correlates with improved survival and a reduced recurrence of CRC [28, 31-33]. Galon et al. were able to demonstrate that type, density, and location of T-lymphocytes represent a more accurate predictor of survival than the widely used UICC-TNM classification [32]. Moreover, tumor associated macrophages, which are associated with poorer outcome in several other tumor entities, predominantly correlate with improved survival in CRC [28, 34-36].

Evidence for a synergistic effect of PD-1 immune checkpoint blockade and NSAID-mediated antitumor pathways has been provided by Zelenay et al. [21, 24]. These data show a pivotal role of $\mathrm{PGE}_{2}$ in the context of antitumor immune surveillance (Figure 1). It was also shown that association of aspirin use with CRC survival is stronger in patients with tumors expressing a low PD1 ligand 
84 expression level. These findings suggest a differential antitumor effect of aspirin according to 86

immune checkpoint status [11].

\section{CRC}

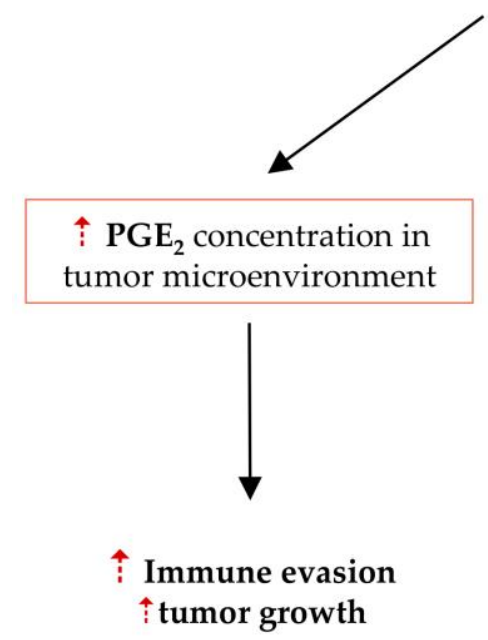

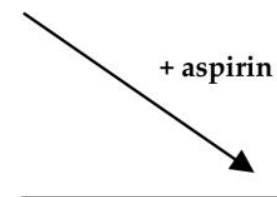
- $\mathrm{PGE}_{2}$ concentration in tumor microenvironment

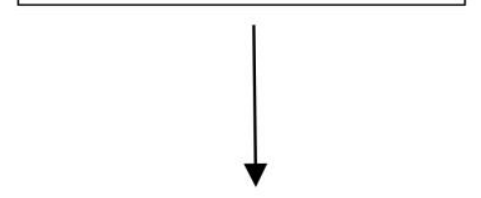

\section{$\hat{i}$ Immune surveillance \\ tumor growth}

87

Figure 1. Effect of $\mathrm{PGE}_{2}$ in colorectal cancer. Figure adapted and modified from [21].

\section{Hypothesis}

Omega-3 polyunsaturated fatty acids to enhance immune therapy in colorectal cancer?

Based on their systematic literature review, the World Cancer Research Fund (WCRF) and the American Institute for Cancer Research (AICR) expert panel concluded that high fish or marine n-3 PUFA (EPA and DHA) consumption possibly decreases CRC risk [37, 38]. PUFA are fatty acids, characterized by at least two carbon-to-carbon double bonds. Their classification is based on the position of the first double bond, counting from the methyl (omega) end [39, 40]. Thus, in omega-3 (n-3) PUFA, the first double bond is located at the third carbon atom, whereas in n-6 PUFA it is at the sixth carbon atom [41, 42]. Eicosapentaenoic acid (EPA; C20:5n-3) and docosahexaenoic acid (DHA; C22:6n-3) are essential members of the n-3 series [43, 44]. Likewise, arachidonic acid (AA; C20:4n-6) has been found to be a crucial member of the $n-6$ series $[45,46]$. A recently published meta-analysis including 19 case-control and 22 prospective cohort studies was able to conclude that intake of fish high in n-3 PUFA may significantly reduce the risk of CRC by as much as $12 \%$ [47]. Additionally, Hall and Kojima et al. observed an inverse correlation between n-3 PUFA blood levels and risk of CRC $[48,49]$.

Several clinical, animal, and in vitro studies have demonstrated the possible preventive and therapeutic role of n-3 PUFA against CRC [50] Thus, it was demonstrated that dietary supplementation with fish oil or EPA significantly reduced crypt cell proliferation and increased apoptosis in patients with colorectal adenomas [51,52]. Additionally, the fat- 1 transgenic mice model provides evidence that n-3 PUFA and their metabolites are likely to suppress colitis-associated 
112 cancer development [53-56]. These mice carry a transferred gene, which encodes for a fatty-acid 113 desaturase enzyme that converts n-6 to n-3 PUFA, resulting in a low n-6 to n-3 ratio of almost 1 [53]. 114 In a study with dextran-sodium-sulfate-induced colitis-associated colon cancer, fat-1 mice showed a 115 reduced number of colonic adenocarcinomas, elevated apoptosis, and enhanced ability to resolve 116 chronic colitis [55]. An animal model using mice with an APC gene defect (Apc ${ }^{\mathrm{Min} /+}$ mice) reported 117 similar results for EPA [57]. Moreover, several in vitro experiments conducted with CRC cell lines 118 demonstrated anti-proliferative effects of EPA and DHA [58-61].

119 These studies have also elucidated the multiple molecular pathways by how n-3 PUFA may 120 modulate cancer development. Previous reviews by us and others summarized and critically 121 analyzed these pathways [50, 62-64]. Several of the effects associated with n-3 PUFA, such as EPA 122 and DHA, as well as n-6 PUFA (e.g. AA) are believed to be mediated through action of lipid 123 mediators [44, 65-69]. AA-derived PGE2, in particular, has shown to be pro-tumorigenic as well as 124 instrumental in tumor immune evasion [13, 14, 21]. Moreover, in a previous study we demonstrated 125 the ability of DHA to reduce proliferation as well as formation of $\mathrm{PGE}_{2}$ in CRC cells [70].

127 Many observations point towards polyunsaturated fatty acids (PUFA) modulating the immune 128 response in the context of colon cancer, possibly mediated by their lipid mediators. These are 129 synthesized through several enzymatic pathways, including COX, lipoxygenase (LOX), and 130 cytochrome P-450 (CYP) monooxygenase pathways [71-73]. Past decades have seen a great number 131 of studies on the functions of AA-derived prostaglandins (PGs), leukotrienes (LTs), lipoxins (LXs), 132 and thromboxanes ( $\mathrm{TXs}$ ) [74]. This interest is largely due to the well-established role of these 133 metabolites in several pathological processes, including inflammatory disorders, cellular 134 proliferation and thrombosis [69, 75].

135 The n-6 PUFA AA promotes a predominantly pro-inflammatory state, whereas EPA and DHA exert 136 a modulating influence on immune cells $[75,76]$. Indeed, AA-derived LTs and PGs can act as potent 137 pro-inflammatory lipid metabolites (depending on cell type and receptor) [75, 77-81]. EPA and 138 DHA, on the other hand, inhibit synthesis of AA-derived, pro-inflammatory eicosanoids such as 139 PGE2 [82]. Moreover, n-3 PUFA are also precursors to anti-inflammatory lipid mediators, such as 140 resolvins (RVs) and protectins (PDs) (Figure 2) [42, 76, 77, 83]. In this context, n-3 PUFA and their 141 derivatives have shown to decrease activation of nuclear factor kappa B (NFкB), a major 142 transcription factor for the upregulation of genes involved in the inflammatory process [46, 84-86]. 143 Activation of NF- $\kappa \mathrm{B}$ results in the secretion of pro-inflammatory cytokines (e.g., IL-1, IL-2, IL-6, 144 IL-12, and TNF- $\alpha$ ), adhesion molecules, and the expression of inducible enzymes, such as COX-2 145 [76]. N-3-PUFA-supplementation and in vitro studies demonstrated that n-3 PUFA can suppress the 146 secretion of IL-1, IL-2, IL-6, and TNF- $\alpha$ from immune cells [87-92]. EPA- and DHA-derived lipid 147 mediators, such as RVs, PDs, and maresins (MaRs) as well as 18-HEPE and 17-HDHA can reduce 148 inflammatory parameters in animal and in vitro studies [66, 93-98]. 
150

151

152

153

154

155

156

157

158

159

160

161

162

163

164

165

166

167

168

169

170

171

172

173

174

175

176

177

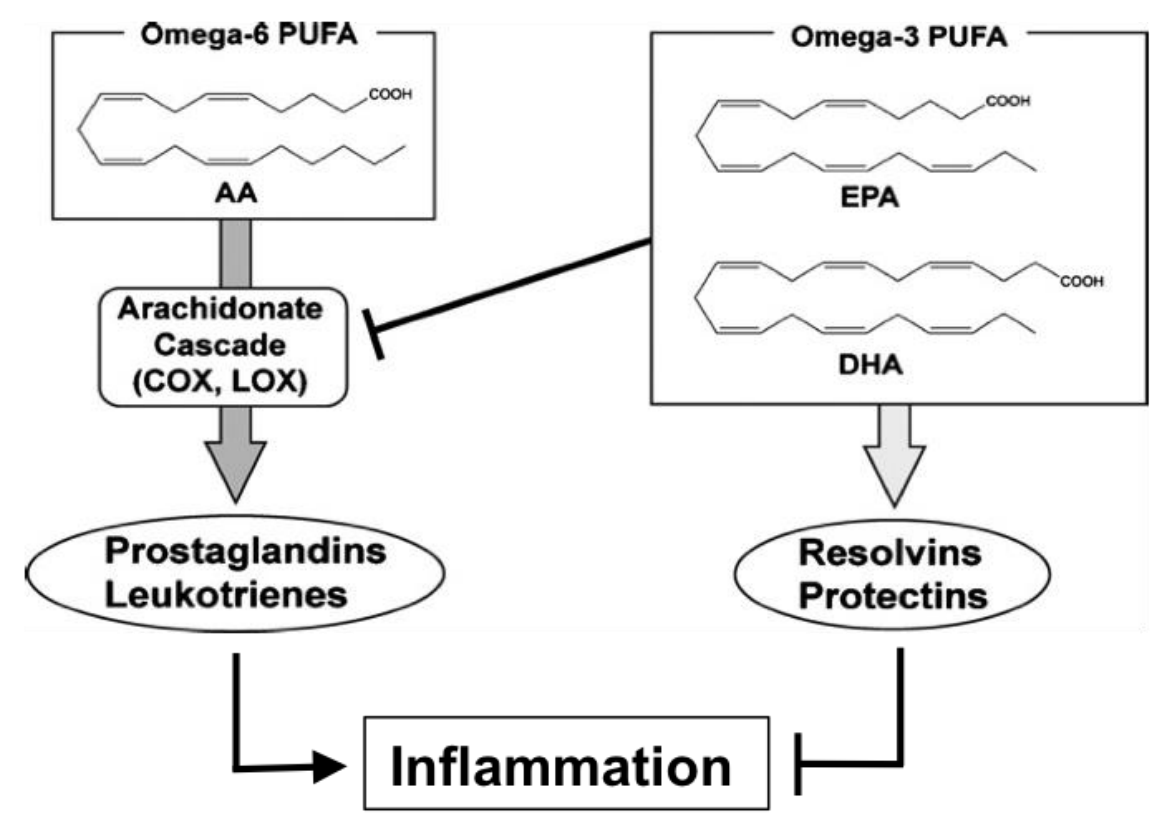

Figure 2. Possible mechanisms of PUFA pro- and anti-inflammatory actions. N-3 PUFA prevent the conversion of AA into pro-inflammatory eicosanoids, such as AA-derived PGs and LTs. In addition, EPA and DHA are precursors to potent anti-inflammatory lipid mediators, such as resolvins and protectins. Figure adapted and modified from [78, 83].

Due to the demonstrated effects of n-3 and n-6 PUFA on the immune system, these fatty acids could affect the immune response to tumors. Particularly in light of the previously published findings of immune-based therapies in CRC patients, and the paradigm of immune-activation as anti-cancer treatment approach, it is now pertinent to reassess the possible effects of n-3 and n-6 PUFA on immune cell activity in this context: Do these fatty acids suppress immune function including anti-tumor immune reactions, or could they even have immune-stimulatory effects in the tumor microenvironment, supporting Cancer Immune Therapy?

As outlined above, high levels of $\mathrm{PGE}_{2}$ play an important role in mediating effects of AA [80, 99-101]. PGE2 inhibits phagocytosis and the TLR-dependent activation of TNF- $\alpha$ secretion via the IL-1Rassociated kinase-M [100-106]. A decrease in PGE2 therefore seems to be a plausible mechanism for immune-modulating effects of DHA and EPA (Figure 2). In line with the possibility to transfer the anti-tumor paradigm stated by Zelenay et al. [21] for aspirin to the n-3 PUFA, an increase in the dietary n-3 to n-6 PUFA ratio was found to not only correlate with higher TNF- $\alpha$ secretion but also with significantly lower levels of AA-derived PGE2 in immune cells [102] as well as colon cancer cells [70]. Indeed, several studies with n-3 PUFA emphasize their inhibitory action on the synthesis of PGE2 [102, 103, 107-109], supporting the hypothesis of increased tumor immune surveillance due to this PGE2-suppressive n-3 PUFA effect.

Similarly, incubation of murine peritoneal macrophages with AA potently inhibited LPS-induced TNF- $\alpha$ production [110]. It was observed that concomitant treatment of these macrophages with AA and indomethacin (inhibiting the synthesis of PGs) restored $90 \%$ of the TNF- $\alpha$ concentration, which indicates that AA exerts an inhibitory effect on TNF- $\alpha$ secretion via increased PG-levels. 
178

179

180

181

182

183

184

185

186

187

188

189

190

191

192

193

In our hands, to directly test for effects of n-3 and n-6 PUFA on differentially induced cytokine secretion by human PBMCs we incubated cells derived from healthy donors with two different stimuli, (1) LPS to mimic activation by bacterial products and (2) colon tumor cell conditioned media to mimic activation by tumor cells (Figure 3). This small study showed that DHA significantly reduced LPS-triggered IL-10 secretion by PBMCs (Figure 3A). Interestingly, DHA had a more pronounced effect on cytokine secretion that was induced by conditioned media derived from human colorectal adenocarcinoma HT-29 cells (CM): CM reduced secretion of IL-10 while increasing TNF- $\alpha$ levels (Figure 3B). The n-6 PUFA AA, on the other hand, reduced TNF- $\alpha$ secretion by PBMCs stimulated with LPS as well as with CM (Figure 3A and B, respectively). In analysis of variance (ANOVA), when compared to PBMCs treated with AA, LPS- as well as CM-induced TNF- $\alpha$ secretion was significantly higher in cells incubated with EPA or DHA $(\mathrm{p}<0.05)$. TNF- $\alpha$ is a typical pro-inflammatory cytokine, while IL-10 has been shown to exert effects limiting cytotoxic T-cell action [111-118]. Considering these findings, our results suggest that incubation of PBMCs with DHA results in a more aggressive immunological response against tumor cells, while AA could be associated with an immunosuppressive effect in the context of tumor immunity.
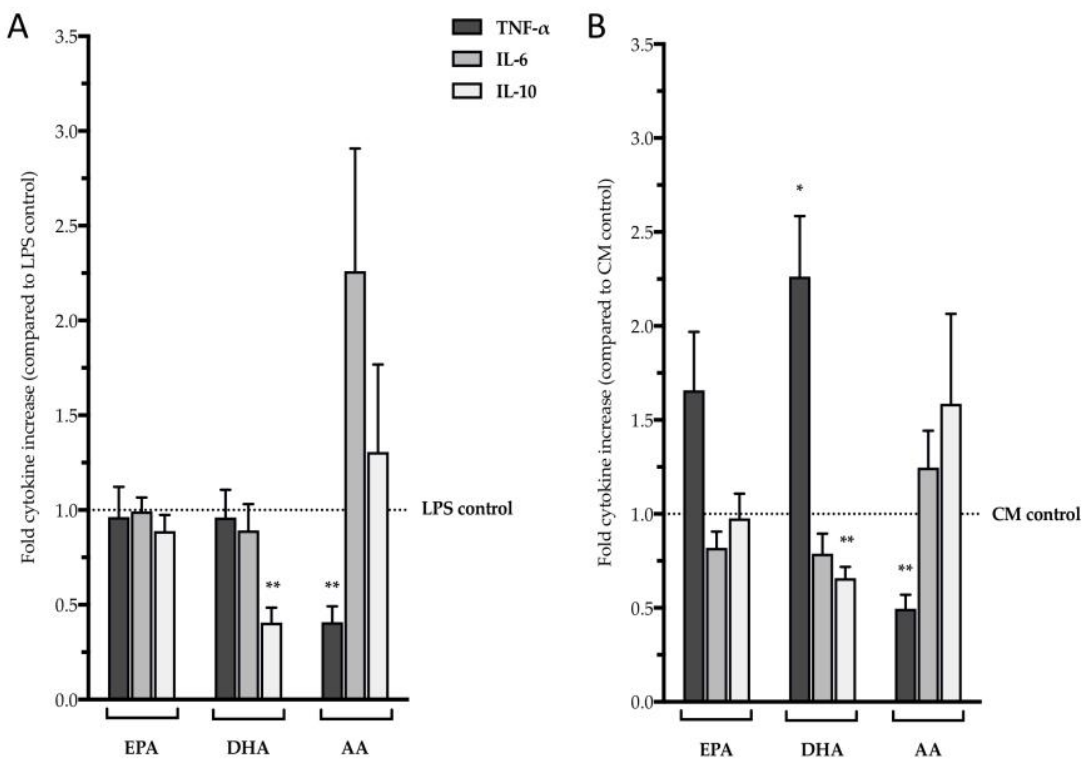

Figure 3. Effect of three major n-3 and n-6 PUFA (EPA, DHA, and AA) on cytokine secretion by PBMCs. (a) LPS-induced cytokine secretion; (b) Cytokine secretion induced by conditioned media derived from human colorectal adenocarcinoma HT-29 cells (CM). This small experimental series was established to explore the immunomodulatory effects of n-3 and n-6 PUFA with regard to a possible effect of CRC tumor cells. For this, PBMCs were isolated from leukocyte depletion filters, acquired from adult blood bank donors. After incubation with EPA, DHA, or AA, PBMCs were stimulated with LPS (a) or CM (b). Subsequently TNF- $\alpha$, IL-6, and IL-10 secretion was measured using ELISA. For controls PBMCs stimulated with LPS or CM, without prior incubation with PUFA, were used (for a detailed description of materials and methods used, refer to supplementary data). Data is expressed as the relative mean + SEM of 5 PBMC donors as compared to LPS and CM control, respectively. ${ }^{*} \mathrm{p}<0.05,{ }^{* *} \mathrm{p}<0.01$.

However, the implications of the observed changes in cytokine secretion are not entirely clear for several reasons. For one, TNF- $\alpha$ has shown to exert ambivalent effects on cancer cells, depending on the activation of intracellular pathways $[119,120]$. Also the role of IL-10 in the context of cancer is 
controversial: While many data show that Il-10 can reduce antigen-specific T-cell activation and induce T-cell anergy $[118,121]$ and might thus be a pro-tumorigenic inflammatory mediator [122], recent data demonstrate an important role for IL-10 in effective immune surveillance of tumor cells $[123,124]$.

\section{Conclusion and Outlook}

The primary prevention of CRC by long-term NSAID-intake, in particular aspirin, is believed to be caused by a reduced conversion of AA into biologically active eicosanoids such as PGE2, which has been shown to contribute to immune evasion by tumor cells [15, 17-21]. Additionally, the recently published studies on immune checkpoint inhibitors demonstrate the clinical effectiveness of increasing an anti-tumor immune response as a novel treatment approach in CRC [123-126]. Indeed, the data reviewed above indicate a possible supporting effect of aspirin in the context of immune checkpoint inhibitor use for cancer therapy [21].

Our summary presented here raises the possibility of a pro-immunogenic effect of n-3 PUFA in the context of the immune system's response to cancer (Figure 4). This could be of particular interest with the advent of immune checkpoint inhibitor therapy in oncology as this implies the possibility of an enhancing effect of n-3 PUFA in the context of these therapeutic interventions.

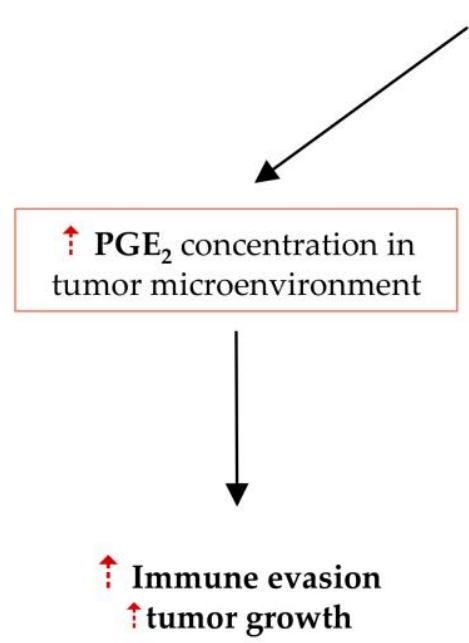

\section{CRC}
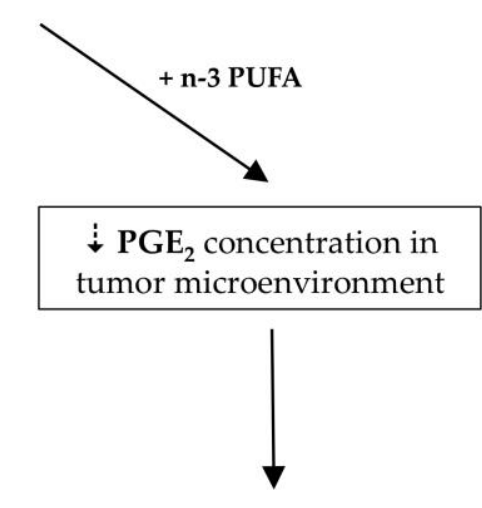

$\uparrow$ Immune surveillance?

tumor growth?

Figure 4. Possible effect of n-3 PUFA in colorectal cancer. In analogy to the aspirin effect increasing the anti-tumor immune response

We therefore propose future studies, in experimental (animal) models as well as in the clinical setting, to test for an enhanced anti-tumor effect of the combination of high n-3 PUFA supplementation with cancer immunotherapy as compared to immunotherapy in the context of a 


\section{$\underline{\text { References }}$}

237 1. Center, M.M., et al., Worldwide Variations in Colorectal Cancer. CA: A Cancer Journal for 238 Clinicians, 2009. 59(6): p. 366-378.

239 2. Ferlay, J., et al., Estimates of worldwide burden of cancer in 2008: GLOBOCAN 2008. Int J Cancer, $240 \quad$ 2010. 127(12): p. 2893-917.

241 3. Algra, A.M. and P.M. Rothwell, Effects of regular aspirin on long-term cancer incidence and 242 metastasis: a systematic comparison of evidence from observational studies versus randomised trials. Lancet 243 Oncol, 2012. 13(5): p. 518-27.

244 4. Benson, A.B., 3rd, Epidemiology, disease progression, and economic burden of colorectal cancer. J 245 Manag Care Pharm, 2007. 13(6 Suppl C): p. S5-18.

246 5. Andersen, V. and U. Vogel, Systematic review: interactions between aspirin, and other nonsteroidal 247 anti-inflammatory drugs, and polymorphisms in relation to colorectal cancer. Aliment Pharmacol Ther, $248 \quad$ 2014. 40(2): p. 147-59.

249 6. Flossmann, E. and P.M. Rothwell, Effect of aspirin on long-term risk of colorectal cancer: consistent 250 evidence from randomised and observational studies. Lancet, 2007. 369(9573): p. 1603-13.

251 7. Gottschall, H., et al., Aspirin alone and combined with a statin suppresses eicosanoid formation in 252 human colon tissue. J Lipid Res, 2018.

253 8. Rothwell, P.M., et al., Long-term effect of aspirin on colorectal cancer incidence and mortality: 20-year 254 follow-up of five randomised trials. Lancet, 2010. 376(9754): p. 1741-50.

255 9. Li, P., et al., Aspirin use after diagnosis but not prediagnosis improves established colorectal cancer 256 survival: a meta-analysis. Gut, 2015. 64(9): p. 1419-25.

257 10. Chan, A.T., et al., Aspirin in the chemoprevention of colorectal neoplasia: an overview. Cancer Prev 258 Res (Phila), 2012. 5(2): p. 164-78.

259 11. Hamada, T., et al., Aspirin Use and Colorectal Cancer Survival According to Tumor CD274 260 (Programmed Cell Death 1 Ligand 1) Expression Status. J Clin Oncol, 2017. 35(16): p. 1836-1844.

261 12. Boland, P.M. and W.W. Ma, Immunotherapy for Colorectal Cancer. Cancers (Basel), 2017. 9(5).

262 13. Wang, D. and R.N. DuBois, An inflammatory mediator, prostaglandin E2, in colorectal cancer. Cancer 263 J, 2013. 19(6): p. 502-10.

264 14. Wang, D. and R.N. Dubois, Prostaglandins and cancer. Gut, 2006. 55(1): p. 115-22.

265 15. Rigas, B., I.S. Goldman, and L. Levine, Altered eicosanoid levels in human colon cancer. J Lab Clin 266 Med, 1993. 122(5): p. 518-23.

267 16. Frommel, T.O., et al., Effect of aspirin on prostaglandin E2 and leukotriene B4 production in human 268 colonic mucosa from cancer patients. Clin Cancer Res, 1997. 3(2): p. 209-13.

269 17. Eberhart, C.E., et al., Up-regulation of cyclooxygenase 2 gene expression in human colorectal adenomas 270 and adenocarcinomas. Gastroenterology, 1994. 107(4): p. 1183-8. 
271 18. Cathcart, M.C., et al., COX-derived prostanoid pathways in gastrointestinal cancer development and 272 progression: novel targets for prevention and intervention. Biochim Biophys Acta, 2012. 1825(1): p. 49-63.

273 19. Azrad, M., C. Turgeon, and W. Demark-Wahnefried, Current evidence linking polyunsaturated 274 Fatty acids with cancer risk and progression. Front Oncol, 2013. 3: p. 224.

275 20. Stolina, M., et al., Specific inhibition of cyclooxygenase 2 restores antitumor reactivity by altering the 276 balance of IL-10 and IL-12 synthesis. J Immunol, 2000. 164(1): p. 361-70.

277 21. Zelenay, S., et al., Cyclooxygenase-Dependent Tumor Growth through Evasion of Immunity. Cell, 278 2015. 162(6): p. 1257-70.

279 22. Le, D.T., et al., PD-1 Blockade in Tumors with Mismatch-Repair Deficiency. N Engl J Med, 2015. $280 \quad 372(26)$ : p. 2509-20.

281 23. Topalian, S.L., et al., Safety, activity, and immune correlates of anti-PD-1 antibody in cancer. N Engl J 282 Med, 2012. 366(26): p. 2443-54.

283 24. Xiao, Y. and G.J. Freeman, The microsatellite instable subset of colorectal cancer is a particularly good 284 candidate for checkpoint blockade immunotherapy. Cancer Discov, 2015. 5(1): p. 16-8.

285 25. Llosa, N.J., et al., The vigorous immune microenvironment of microsatellite instable colon cancer is 286 balanced by multiple counter-inhibitory checkpoints. Cancer Discov, 2015. 5(1): p. 43-51.

287 26. Atreya, I. and M.F. Neurath, Immune cells in colorectal cancer: prognostic relevance and therapeutic 288 strategies. Expert Rev Anticancer Ther, 2008. 8(4): p. 561-72.

289 27. Di Caro, G., et al., Immune cells: plastic players along colorectal cancer progression. J Cell Mol Med, 290 2013. 17(9): p. 1088-95.

291 28. Roxburgh, C.S. and D.C. McMillan, The role of the in situ local inflammatory response in predicting 292 recurrence and survival in patients with primary operable colorectal cancer. Cancer Treat Rev, 2012. 38(5): 293 p. 451-66.

294 29. Mei, Z., et al., Tumour-infiltrating inflammation and prognosis in colorectal cancer: systematic review 295 and meta-analysis. Br J Cancer, 2014. 110(6): p. 1595-605.

296 30. Richards, C.H., et al., The clinical utility of the local inflammatory response in colorectal cancer. Eur J 297 Cancer, 2014. 50(2): p. 309-19.

298 31. Pages, F., et al., In situ cytotoxic and memory T cells predict outcome in patients with early-stage 299 colorectal cancer. J Clin Oncol, 2009. 27(35): p. 5944-51.

300 32. Galon, J., et al., Type, density, and location of immune cells within human colorectal tumors predict 301 clinical outcome. Science, 2006. 313(5795): p. 1960-4.

302 33. Jochems, C. and J. Schlom, Tumor-infiltrating immune cells and prognosis: the potential link between 303 conventional cancer therapy and immunity. Experimental biology and medicine, 2011. 236(5): p. 567-79.

304 34. Ong, S.M., et al., Macrophages in human colorectal cancer are pro-inflammatory and prime T cells 305 towards an anti-tumour type-1 inflammatory response. Eur J Immunol, 2012. 42(1): p. 89-100.

306 35. Sica, A., et al., Macrophage polarization in tumour progression. Seminars in cancer biology, 2008. 307 18(5): p. 349-55. 
308 36. Zhang, Q.W., et al., Prognostic significance of tumor-associated macrophages in solid tumor: a 309 meta-analysis of the literature. PLoS One, 2012. 7(12): p. e50946.

310 37. Norat, T.C., D; Lau, D; Aune, D; Vieira, R, WCRF/AICR Systematic Literature Review Continuous

311 Update Project Report - The Associations between Food, Nutrition and Physical Activity and the Risk of 312 Colorectal Cancer. 2010.

313 38. WCRF/AICR, Food, Nutrition, Physical activity, and the Prevention of Cancer: a Global Perspective. 314 American Institute of Cancer Research (AICR), Washington, DC, 2007.

315 39. Wallis, J.G., J.L. Watts, and J. Browse, Polyunsaturated fatty acid synthesis: what will they think of 316 next? Trends Biochem Sci, 2002. 27(9): p. 467.

317 40. Sassa, T. and A. Kihara, Metabolism of Very Long-Chain Fatty Acids: Genes and Pathophysiology. 318 Biomol Ther (Seoul), 2014. 22(2): p. 83-92.

319 41. Abedi, E. and M.A. Sahari, Long-chain polyunsaturated fatty acid sources and evaluation of their 320 nutritional and functional properties. Food Sci Nutr, 2014. 2(5): p. 443-463.

321 42. Weylandt, K.H., et al., Omega-3 fatty acids and their lipid mediators: towards an understanding of 322 resolvin and protectin formation. Prostaglandins Other Lipid Mediat, 2012. 97(3-4): p. 73-82.

323 43. Massaro, M., et al., Basic mechanisms behind the effects of $n-3$ fatty acids on cardiovascular disease. 324 Prostaglandins Leukot Essent Fatty Acids, 2008. 79(3-5): p. 109-15.

325 44. Tvrzicka, E., et al., Fatty acids as biocompounds: their role in human metabolism, health and disease - a 326 review. part 1: classification, dietary sources and biological functions. Biomedical Papers, 2011. 155(2): p. 327 117-130.

328 45. Harris, W.S., et al., Omega-6 fatty acids and risk for cardiovascular disease: a science advisory from the 329 American Heart Association Nutrition Subcommittee of the Council on Nutrition, Physical Activity, and 330 Metabolism; Council on Cardiovascular Nursing; and Council on Epidemiology and Prevention. Circulation, 331 2009. 119(6): p. 902-7.

332 46. Calder, P.C., Marine omega-3 fatty acids and inflammatory processes: Effects, mechanisms and clinical 333 relevance. Biochim Biophys Acta, 2014.

334 47. Wu, S., et al., Fish consumption and colorectal cancer risk in humans: a systematic review and 335 meta-analysis. Am J Med, 2012. 125(6): p. 551-9.e5.

336 48. Hall, M.N., et al., Blood levels of long-chain polyunsaturated fatty acids, aspirin, and the risk of 337 colorectal cancer. Cancer Epidemiol Biomarkers Prev, 2007. 16(2): p. 314-21.

338 49. Kojima, M., et al., Serum levels of polyunsaturated fatty acids and risk of colorectal cancer: a prospective 339 study. Am J Epidemiol, 2005. 161(5): p. 462-71.

340 50. Weylandt, K.H., et al., Omega-3 Polyunsaturated Fatty Acids: The Way Forward in Times of Mixed 341 Evidence. Biomed Res Int, 2015. 2015: p. 143109.

342 51. Courtney, E.D., et al., Eicosapentaenoic acid (EPA) reduces crypt cell proliferation and increases 343 apoptosis in normal colonic mucosa in subjects with a history of colorectal adenomas. International journal of 344 colorectal disease, 2007. 22(7): p. 765-76. 
52. Anti, M., et al., Effects of different doses of fish oil on rectal cell proliferation in patients with sporadic colonic adenomas. Gastroenterology, 1994. 107(6): p. 1709-18.

53. Kang, J.X., et al., Transgenic mice: fat-1 mice convert $n-6$ to $n-3$ fatty acids. Nature, 2004. 427(6974): p. 504.

54. Gleissman, H., J.I. Johnsen, and P. Kogner, Omega-3 fatty acids in cancer, the protectors of good and the killers of evil? Exp Cell Res, 2010. 316(8): p. 1365-73.

351 55. Jia, Q., et al., Reduced colitis-associated colon cancer in Fat-1 ( $n-3$ fatty acid desaturase) transgenic mice. 352 Cancer Res, 2008. 68(10): p. 3985-91.

353 56. Nowak, J., et al., Colitis-associated colon tumorigenesis is suppressed in transgenic mice rich in 354 endogenous n-3 fatty acids. Carcinogenesis, 2007. 28(9): p. 1991-5.

355 57. Petrik, M.B., et al., Highly unsaturated (n-3) fatty acids, but not alpha-linolenic, conjugated linoleic or gamma-linolenic acids, reduce tumorigenesis in Apc(Min/+) mice. J Nutr, 2000. 130(10): p. 2434-43.

58. Benais-Pont, G., et al., Omega-3 polyunsaturated fatty acids and ionizing radiation: combined cytotoxicity on human colorectal adenocarcinoma cells. Nutrition, 2006. 22(9): p. 931-9.

59. Zhang, C., et al., Growth Inhibitory Effect of Polyunsaturated Fatty Acids (PUFAs) on Colon Cancer Cells via Their Growth Inhibitory Metabolites and Fatty Acid Composition Changes. PLoS One, 2015. 10(4): p. e0123256.

60. Dommels, Y.E., et al., The role of cyclooxygenase in n-6 and n-3 polyunsaturated fatty acid mediated effects on cell proliferation, PGE(2) synthesis and cytotoxicity in human colorectal carcinoma cell lines. Carcinogenesis, 2003. 24(3): p. 385-92.

61. Schonberg, S.A., et al., Closely related colon cancer cell lines display different sensitivity to polyunsaturated fatty acids, accumulate different lipid classes and downregulate sterol regulatory element-binding protein 1. Febs j, 2006. 273(12): p. 2749-65.

62. Serini, S., E. Piccioni, and G. Calviello, $\omega-3$ PUFAs and Colon Cancer: Experimental Studies and Human Interventional Trials, in Dietary Omega-3 Polyunsaturated Fatty Acids and Cancer, G. Calviello and S. Serini, Editors. 2010, Springer Netherlands: Dordrecht. p. 67-89.

371 63. Serini, S., et al., Differential anti-cancer effects of purified EPA and DHA and possible mechanisms involved. Curr Med Chem, 2011. 18(26): p. 4065-75.

373 64. Gabriella, C., S. Simona, and P. Paola, n-3 Polyunsaturated Fatty Acids as Signal Transduction 374 Modulators and Therapeutical Agents in Cancer. Current Signal Transduction Therapy, 2006. 1(3): p. $375 \quad 255-271$.

65. Astarita, G., et al., Targeted lipidomics strategies for oxygenated metabolites of polyunsaturated fatty acids. Biochim Biophys Acta, 2014.

378 66. Wang, W., et al., omega-3 polyunsaturated fatty acids-derived lipid metabolites on angiogenesis, 379 inflammation and cancer. Prostaglandins Other Lipid Mediat, 2014. 113-115: p. 13-20.

380 67. Powell, W.S. and J. Rokach, Biosynthesis, biological effects, and receptors of hydroxyeicosatetraenoic 381 acids (HETEs) and oxoeicosatetraenoic acids (oxo-ETEs) derived from arachidonic acid. Biochim Biophys 382 Acta, 2014. 
383 68. Stables, M.J. and D.W. Gilroy, Old and new generation lipid mediators in acute inflammation and resolution. Prog Lipid Res, 2011. 50(1): p. 35-51.

69. Calder, P.C., Dietary arachidonic acid: harmful, harmless or helpful? Br J Nutr, 2007. 98(3): p. 451-3.

70. Habbel, P., et al., Docosahexaenoic acid suppresses arachidonic acid-induced proliferation of LS-174T human colon carcinoma cells. World J Gastroenterol, 2009. 15(9): p. 1079-84.

71. Lagarde, M., et al., Lipidomics of essential fatty acids and oxygenated metabolites. Mol Nutr Food Res, 2013. 57(8): p. 1347-58.

72. Nicolaou, A., et al., Polyunsaturated Fatty Acid-derived lipid mediators and T cell function. Front Immunol, 2014. 5: p. 75.

73. Arita, M., Mediator lipidomics in acute inflammation and resolution. J Biochem, 2012. 152(4): p. 313-9.

74. Weylandt, K.H. and J.X. Kang, Rethinking lipid mediators. Lancet, 2005. 366(9486): p. 618-20.

75. Schmitz, G. and J. Ecker, The opposing effects of n-3 and n-6 fatty acids. Prog Lipid Res, 2008. 47(2): p. 147-55.

76. Patterson, E., et al., Health implications of high dietary omega-6 polyunsaturated Fatty acids. J Nutr Metab, 2012. 2012: p. 539426.

398 77. Calder, P.C., Dietary modification of inflammation with lipids. Proc Nutr Soc, 2002. 61(3): p. 345-58.

399 78. Isobe, Y. and M. Arita, Identification of novel omega-3 fatty acid-derived bioactive metabolites based on 400 a targeted lipidomics approach. J Clin Biochem Nutr, 2014. 55(2): p. 79-84.

401 79. Sharma, J.N. and L.A. Mohammed, The role of leukotrienes in the pathophysiology of inflammatory 402 disorders: is there a case for revisiting leukotrienes as therapeutic targets? Inflammopharmacology, 2006. 403 14(1-2): p. 10-6.

404 80. Tilley, S.L., T.M. Coffman, and B.H. Koller, Mixed messages: modulation of inflammation and 405 immune responses by prostaglandins and thromboxanes. J Clin Invest, 2001. 108(1): p. 15-23.

81. Jandl, K., et al., Activated prostaglandin D2 receptors on macrophages enhance neutrophil recruitment into the lung. J Allergy Clin Immunol, 2016. 137(3): p. 833-43. IUBMB Life, 2015. 67(9): p. 659-67.

83. Seki, H., Y. Tani, and M. Arita, Omega-3 PUFA derived anti-inflammatory lipid mediator resolvin E1.

412 84. Calviello, G., et al., Experimental evidence of omega-3 polyunsaturated fatty acid modulation of 413 inflammatory cytokines and bioactive lipid mediators: their potential role in inflammatory, neurodegenerative, 414 and neoplastic diseases. Biomed Res Int, 2013. 2013: p. 743171.

415 85. Hudert, C.A., et al., Transgenic mice rich in endogenous omega-3 fatty acids are protected from colitis. 416 Proc Natl Acad Sci U S A, 2006. 103(30): p. 11276-81.

417 86. Novak, T.E., et al., NF- $\kappa B$ inhibition by $\omega-3$ fatty acids modulates LPS-stimulated macrophage TNF- $\alpha$ 418 transcription. Vol. 284. 2003. L84-L89. 
87. Novak, T.E., et al., NF-kappa B inhibition by omega -3 fatty acids modulates LPS-stimulated macrophage TNF-alpha transcription. American journal of physiology. Lung cellular and molecular physiology, 2003. 284(1): p. L84-9.

88. Purasiri, P., et al., Modulation in vitro of human natural cytotoxicity, lymphocyte proliferative response to mitogens and cytokine production by essential fatty acids. Immunology, 1997. 92(2): p. 166-72.

89. Endres, S., et al., Dietary supplementation with n-3 fatty acids suppresses interleukin-2 production and mononuclear cell proliferation. J Leukoc Biol, 1993. 54(6): p. 599-603.

90. Kumar, G.S., et al., Effect of n-6 and n-3 fatty acids on the proliferation of human lymphocytes and their secretion of TNF- $\alpha$ and IL-2 in vitro. Nutrition Research, 1992. 12(7): p. 815-823.

91. Meydani, S.N., et al., Oral (n-3) fatty acid supplementation suppresses cytokine production and lymphocyte proliferation: comparison between young and older women. J Nutr, 1991. 121(4): p. 547-55.

92. Blok WL, K.M., van der Meer JW., Modulation of Inflammation and Cytokine Production by Dietary (n-3) Faty Acids. J Nutr. Jun;:, 1996 126: p. 1515-33.

93. Bento, A.F., et al., Omega-3 fatty acid-derived mediators 17(R)-hydroxy docosahexaenoic acid, aspirin-triggered resolvin D1 and resolvin D2 prevent experimental colitis in mice. J Immunol, 2011. 187(4): p. 1957-69.

94. Krishnamurthy, V.R., et al., Total Synthesis and Bioactivity of 18(R)-Hydroxyeicosapentaenoic Acid. J Org Chem, 2011. 76(13): p. 5433-7.

95. Weylandt, K.H., et al., Suppressed liver tumorigenesis in fat-1 mice with elevated omega-3 fatty acids is associated with increased omega-3 derived lipid mediators and reduced TNF-alpha. Carcinogenesis, 2011. 32(6): p. 897-903.

96. Serhan, C.N., et al., Anti-inflammatory actions of neuroprotectin D1/protectin D1 and its natural stereoisomers: assignments of dihydroxy-containing docosatrienes. J Immunol, 2006. 176(3): p. 1848-59.

97. Serhan, C.N., et al., Maresins: novel macrophage mediators with potent antiinflammatory and proresolving actions. J Exp Med, 2009. 206(1): p. 15-23.

98. Serhan, C.N., et al., Protectins and maresins: New pro-resolving families of mediators in acute inflammation and resolution bioactive metabolome. Biochim Biophys Acta, 2015. 1851(4): p. 397-413.

99. Aronoff, D.M., C. Canetti, and M. Peters-Golden, Prostaglandin E2 inhibits alveolar macrophage phagocytosis through an E-prostanoid 2 receptor-mediated increase in intracellular cyclic AMP. J Immunol, 2004. 173(1): p. 559-65.

100. Domingo-Gonzalez, R., et al., Prostaglandin E2-induced changes in alveolar macrophage scavenger receptor profiles differentially alter phagocytosis of Pseudomonas aeruginosa and Staphylococcus aureus post-bone marrow transplant. J Immunol, 2013. 190(11): p. 5809-17.

101. Hubbard, L.L., et al., A role for IL-1 receptor-associated kinase-M in prostaglandin E2-induced immunosuppression post-bone marrow transplantation. J Immunol, 2010. 184(11): p. 6299-308.

102. Hardardottir, I. and J.E. Kinsella, Increasing the dietary (n-3) to (n-6) polyunsaturated fatty acid ratio increases tumor necrosis factor production by murine resident peritoneal macrophages without an effect on elicited peritoneal macrophages. The Journal of nutrition, 1992. 122(10): p. 1942-51. 
103. Petursdottir, D.H., I. Olafsdottir, and I. Hardardottir, Dietary fish oil increases tumor necrosis factor secretion but decreases interleukin-10 secretion by murine peritoneal macrophages. J Nutr, 2002. 132(12): p. 3740-3.

104. Ertel, W., et al., Modulation of macrophage membrane phospholipids by n-3 polyunsaturated fatty acids increases interleukin 1 release and prevents suppression of cellular immunity following hemorrhagic shock. Arch Surg, 1993. 128(1): p. 15-20; discussion 20-1.

105. Erickson, K.L. and N.E. Hubbard, Dietary fish oil modulation of macrophage tumoricidal activity. Nutrition, 1996. 12(1 Suppl): p. S34-8.

106. Kalinski, P., Regulation of immune responses by prostaglandin E2. J Immunol, 2012. 188(1): p. 21-8.

107. Hegazi, R.A., et al., Dietary fatty acids modulate chronic colitis, colitis-associated colon neoplasia and COX-2 expression in IL-10 knockout mice. Nutrition, 2006. 22(3): p. 275-82.

108. Hardardottir, I. and J.E. Kinsella, Tumor necrosis factor production by murine resident peritoneal macrophages is enhanced by dietary n-3 polyunsaturated fatty acids. Biochimica et biophysica acta, 1991. 1095(3): p. 187-95.

109. Lokesh, B.R., T.J. Sayers, and J.E. Kinsella, Interleukin-1 and tumor necrosis factor synthesis by mouse peritoneal macrophages is enhanced by dietary n-3 polyunsaturated fatty acids. Immunology Letters, 1990. 23(4): p. 281-285.

110. Hubbard, N.E., et al., Effects of in vitro exposure to arachidonic acid on TNF-alpha production by murine peritoneal macrophages. J Leukoc Biol, 1993. 54(2): p. 105-10.

111. Sethi, G., B. Sung, and B.B. Aggarwal, TNF: a master switch for inflammation to cancer. Front Biosci, 2008. 13: p. 5094-107.

112. Tracey, K.J. and A. Cerami, Tumor necrosis factor: a pleiotropic cytokine and therapeutic target. Annu Rev Med, 1994. 45: p. 491-503.

113. Akira, S., et al., Biology of multifunctional cytokines: IL 6 and related molecules (IL 1 and TNF). Faseb j, 1990. 4(11): p. 2860-7.

114. Rizzo, A., et al., Intestinal inflammation and colorectal cancer: A double-edged sword? World J Gastroenterol, 2011. 17(26): p. 3092-100.

115. Ghezzi, P. and A. Cerami, Tumor necrosis factor as a pharmacological target. Mol Biotechnol, 2005. 31(3): p. 239-44.

116. Fiorentino, D.F., et al., IL-10 acts on the antigen-presenting cell to inhibit cytokine production by Th1 cells. J Immunol, 1991. 146(10): p. 3444-51.

117. Barsig, J., et al., Lipopolysaccharide-induced interleukin-10 in mice: role of endogenous tumor necrosis factor-alpha. Eur J Immunol, 1995. 25(10): p. 2888-93.

118. de Waal Malefyt, R., et al., Interleukin 10(IL-10) inhibits cytokine synthesis by human monocytes: an autoregulatory role of IL-10 produced by monocytes. J Exp Med, 1991. 174(5): p. 1209-20.

119. Balkwill, F., Tumour necrosis factor and cancer. Nat Rev Cancer, 2009. 9(5): p. 361-71. 
493 120. Grivennikov, S.I. and M. Karin, Dangerous liaisons: STAT3 and NF-kappaB collaboration and 494 crosstalk in cancer. Cytokine Growth Factor Rev, 2010. 21(1): p. 11-9.

495 121. de Waal Malefyt, R., et al., Interleukin 10 (IL-10) and viral IL-10 strongly reduce antigen-specific 496 human T cell proliferation by diminishing the antigen-presenting capacity of monocytes via downregulation of 497 class II major histocompatibility complex expression. J Exp Med, 1991. 174(4): p. 915-24.

498 122. Zhang, Q., B. Zhu, and Y. Li, Resolution of Cancer-Promoting Inflammation: A New Approach for 499 Anticancer Therapy. Front Immunol, 2017. 8: p. 71.

500 123. Mumm, J.B., et al., IL-10 elicits IFNgamma-dependent tumor immune surveillance. Cancer Cell, 2011. $501 \quad 20(6):$ p. 781-96.

502 124. Dennis, K.L., et al., Current status of interleukin-10 and regulatory T-cells in cancer. Curr Opin 503 Oncol, 2013. 25(6): p. 637-45. 